
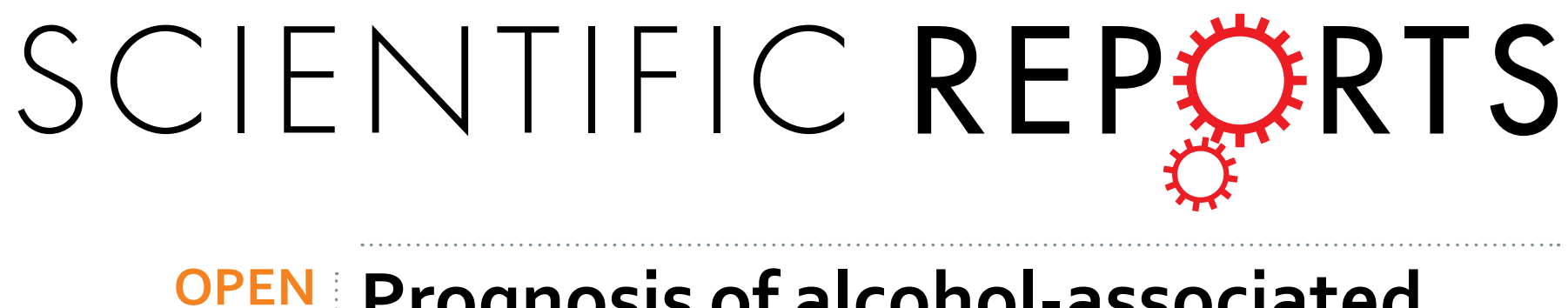

\title{
Prognosis of alcohol-associated lactic acidosis in critically ill patients: an 8-year study
}

Received: 22 February 2016

Accepted: 27 September 2016

Published: 17 October 2016

\section{Chun-Chieh Yang ${ }^{1}$, Khee-Siang Chan ${ }^{1}$, Kuei-Ling Tseng ${ }^{2} \&$ Shih-Feng Weng ${ }^{3}$}

Lactic acidosis is common in critical care; by contrast, a subtype called alcohol-associated lactic acidosis (AALA) is rarely encountered. The primary purpose of this study was to determine the prognosis of AALA in critically ill patients and the second aim was to determine whether the survival was associated to the peak blood lactate concentration. An 8-year retrospective analysis of adult patients admitted to the intensive care unit (ICU) with AALA between January 2007 and December 2014 was considered in a tertiary care hospital. In total, 23 patients were analyzed and the median peak blood lactate level was $15.9 \mathrm{mmol} / \mathrm{L}$. Only 2 patients (8.7\%) presented peak blood lactate levels $<10 \mathrm{mmol} / \mathrm{L}$. In this study, 21 patients survived from ICU and hospital, the mortality rate was $8.7 \%$. The result indicted the survival of AALA was not associated with peak blood lactate concentration although survivors still had a better lactate clearance rate per hour than non-survivors. Moreover, AALA patients with coexisting sepsis presenting higher lactate clearance rate and shorter lactate clearance time than those of AALA patients with solely sepsis-related lactic acidosis.

Lactic acidosis is often encountered in intensive care unit (ICU) and can be associated with a variety of disease states, drugs and toxins including ethanol ingestion ${ }^{1}$. In view of biochemistry of lactate formation, lactic acidosis can be classified into two subtypes: Type A is associated with hypoperfusion or tissue hypoxia, related to imbalance of oxygen delivery and oxygen consumption, while, Type B includes conditions affecting the production and elimination of lactate, unrelated to oxygen debt $^{2,3}$. The potential seriousness of lactic acidosis is its strong association with increased mortality ${ }^{4-9}$. A blood lactate concentration in excess of $5 \mathrm{mmol} / \mathrm{L}$ on ICU admission is associated with a 3 - and 30 -day mortality rate of $59 \%$ and $83 \%$, respectively ${ }^{10}$.

In fact, lactic acid is produced and metabolized in all cells of the body except red blood cells, which have no mitochondria ${ }^{11}$. Review of the formation of lactic acid shows that glucose is broken down to pyruvate through glycolysis, and pyruvate is then converted to lactate as the end product of anaerobic metabolism. On the other hand, lactates can only be metabolized via their conversion back to pyruvate ${ }^{12}$. In addition, ethanol is mainly metabolized in the liver by alcohol dehydrogenase. After being oxidized to acetaldehyde, acetaldehyde is further oxidized to acetic acid by aldehyde dehydrogenase. Both enzymatic steps result in the generation of reduced form of nicotinamide adenine dinucleotide dehydrogenase (NADH). The increase of the NADH/NAD ratio favors the metabolism of pyruvate to lactate; furthermore, the elimination of lactate depends mainly on the conversion of pyruvate to acetyl CoA, gluconeogenesis, and its uptake by the liver and kidneys. This conversion of pyruvate to acetyl CoA requires the enzyme called pyruvate dehydrogenase and the coenzyme called thiamine. Therefore, chronic alcoholism and associated malnutrition may also decrease lactate utilization due to inadequate intake of the water-soluble vitamins such as thiamine and biotin. Theoretically both the metabolism of ethanol and the deficiency of thiamine tend to drive the formation of lactate and subsequently lead to the result of severe lactic acidosis in patients with acute or chronic abuse of alcohol. The primary purpose of this study was to determine the prognosis of alcohol-associated lactic acidosis (AALA) in critically ill adult patients, and the second aim was to verify whether the survival was associated to the peak blood lactate concentration.

\footnotetext{
${ }^{1}$ Department of Intensive Care Medicine, Chi Mei Medical Center, Tainan, Taiwan. ${ }^{2}$ Department of Respiratory Therapy, Chi Mei Medical Center, Tainan, Taiwan. ${ }^{3}$ Department of Healthcare Administration and Medical Informatics, Kaohsiung Medical University, Kaohsiung, Taiwan. Correspondence and requests for materials should
} be addressed to S.-F.G. (email: sfweng@kmu.edu.tw) 


\begin{tabular}{|l|l|}
\hline Variable & Definition \\
\hline Alcohol abuse & Uncontrolled alcohol drinking despite adverse consequences such as hepatic toxicity. \\
\hline Hypoglycemic event & Plasma glucose $<70 \mathrm{mg} / \mathrm{dL}$ with or without symptoms ${ }^{27}$. \\
\hline Acute kidney injury & At least 1.5 times increase in baseline serum creatinine. \\
\hline Chronic kidney disease & Baseline serum creatinine $\geqq 1.5 \mathrm{mg} / \mathrm{dL}$ for men and $\geqq 1.4 \mathrm{mg} / \mathrm{dL}$ for women. \\
\hline Liver disease & $\begin{array}{l}\text { The presence of liver cirrhosis or hepatitis. Cirrhosis is diagnosed according to clinical manifestations, laboratory } \\
\text { results and image studies; and hepatitis is diagnosed using the evidence regarding abnormal hepatic biochemical } \\
\text { and functional tests. }\end{array}$ \\
\hline Sepsis & The presence of infection together with systemic manifestations of infection. \\
\hline High MCV & MCV $>100 \mathrm{fL}$ (normal range of MCV: $80-100 \mathrm{fL})$. \\
\hline Hyperammonemia & Serum ammonia level $>32 \mu \mathrm{mol} / \mathrm{L}$ (normal range of ammonia level: $11-32 \mu \mathrm{mol} / \mathrm{L})$. \\
\hline $\begin{array}{l}\text { Lactate clearance rate } \\
\text { per hour }\end{array}$ & $\begin{array}{l}100 \% \times[(\text { peak blood lactate level }- \text { dynamic blood lactate level when blood lactate concentration }<5 \mathrm{mmol} / \mathrm{L}) / \\
(\text { peak blood lactate level) } /(\text { time interval by hours spent between the peak and dynamic blood lactate level). }\end{array}$ \\
\hline
\end{tabular}

Table 1. Definition of variables.

\section{Methods}

Study sample. This was a retrospective observational study in the ICUs (96 beds) of a tertiary care medical center. Patients who were admitted to the ICU with the diagnosis of AALA between January 2007 and December 2014 were recruited into this study. AALA refers to patients with alcohol abuse and a blood lactate level $>5 \mathrm{mmol} / \mathrm{L}$. Sometimes the diagnosis was considered by the clinicians in charge despite the level of serum alcohol. Patients who had an AALA history before January 2007 were excluded. This study was approved by the Institutional Review Board of Chi Mei Medical Center (IRB-10407-002) and waived the need for informed consent. The methods were carried out in accordance with the approved guidelines.

All clinical and laboratory data at ICU admission and ICU stay were recorded, including: (1) Clinical characteristics: age, gender, Acute Physiology and Chronic Health Evaluation II (APACHE II) score, hypoglycemic event, seizure episode, coexisting diseases such as gastrointestinal (GI) bleeding, sepsis, liver disease (liver cirrhosis, hepatitis), acute kidney injury, chronic kidney disease and the status of ICU and hospital discharge; (2) Laboratory characteristics: peak blood lactate level, dynamic blood lactate level when blood lactate concentration was $<5 \mathrm{mmol} / \mathrm{L}$, time interval by hours between the peak and dynamic blood lactate level, nadir arterial $\mathrm{PH}$ and bicarbonate level, presence of ketone bodies in either urine or blood specimens, serum ethanol level, serum methanol level, presence of high mean corpuscular volume (MCV) of red blood cell, and presence of hyperammonemia; (3) Treatment modalities: during the treatment period of lactic acidosis from blood lactate level $>5 \mathrm{mmol} / \mathrm{L}$ to $<5 \mathrm{mmol} / \mathrm{L}$, the clinical need of vasoactive support, ventilator support, and renal replacement therapy (hemodialysis or continuous veno-venous hemofiltration) was also recorded. All the definitions of variables were listed in Table 1.

Statistical analysis. The clinical characteristics of patients with AALA were both expressed as mean and standard deviation and median with interquartile range (IQR) for continuous variables and percentage frequency for categorical variables. For continuous variables, Wilcoxon rank-sum tests were used to compare the difference between survivor and non-survivor sub-groups due to the small sample sizes. Pearson's chi-square tests were applied to compare the category data between groups. If $20 \%$ of the cells had expected frequencies of less than 5 , Fisher's exact tests was used. The significant level was set at 0.05 for the two sided test. SAS for Windows 9.4 (SAS Institute, Inc, Cary, NC, USA) was performed for all analyses.

\section{Results}

During this study period, twenty-three patients were admitted to the ICU with AALA. The clinical and laboratory characteristics of these patients were shown in Table 2 and most of patients were male $(n=22,22 / 23=95.7 \%)$. The median peak blood lactate level was $15.9 \mathrm{mmol} / \mathrm{L}(\mathrm{IQR}=8.2 \mathrm{mmol} / \mathrm{L})$. There were only 2 patients $(8.7 \%)$ presenting peak blood lactate level $<10 \mathrm{mmol} / \mathrm{L}$; other 21 patients $(91.3 \%)$ were with blood lactate concentration $>10 \mathrm{mmol} / \mathrm{L}$, including 8 patients $>10 \mathrm{but} \leqq 15 \mathrm{mmol} / \mathrm{L}$ as well as 5 patients $>15$ but $\leqq 20 \mathrm{mmol} / \mathrm{L}$. Other 8 patients had a peak blood lactate level of $>20 \mathrm{mmol} / \mathrm{L}$.

Blood or urine ketone body test was performed in 20 patients and $15(75.0 \%)$ of them showed positive results. Serum alcohol level was checked in 14 patients and positive results were found in 10 patients. No patients had seizure episode or methanol intoxication. Most patients presented hyperammonemia (73.9\%) and high MCV (60.9\%). In addition, 16 patients (69.6\%) presented coexisting acute kidney injury while 13 patients (56.5\%) were with liver disease and 11 patients $(47.8 \%)$ had sepsis.

The comparison between the survivors and non-survivors was demonstrated in Table 3 . There were 21 patients survived from the ICU and hospital. The mortality rate in our study was $8.7 \%$. In addition, the survival of AALA was not associated with the peak blood lactate concentration.

\section{Discussion}

This study presented 23 patients admitted to the ICU with AALA in an 8-year period, and 21 patients survived from the ICU and hospital. Between survivors and non-survivors, the peak blood lactate concentration 


\begin{tabular}{|l|c|c|c|}
\hline \multirow{2}{*}{ Characteristics } & \multicolumn{2}{|c|}{ Values (N=23) } \\
\cline { 2 - 4 } & $\mathbf{n}(\%)$ & Median/IRQ & mean \pm SD \\
\hline Age (years) & & $51.0 / 10.0$ & $50.0 \pm 10.9$ \\
\hline APACHE II score & & $14.0 / 9.0$ & $15.5 \pm 6.7$ \\
\hline Peak lactate level (mmol/L) & & $7.25 / 0.34$ & $7.18 \pm 0.21$ \\
\hline Nadir arterial pH & & $8.7 / 11.3$ & $9.5 \pm 5.8$ \\
\hline Nadir arterial bicarbonate level (mmol/L) & & \\
\hline Sex, male, (\%) & $22(95.7 \%)$ & & \\
\hline Positive blood or urine ketone body, $\mathrm{n}(\%)(\mathrm{N}=20)$ & $15\left(75.0 \%{ }^{*}\right)$ & & \\
\hline Positive serum alcohol level, $\mathrm{n}(\%)(\mathrm{N}=14)$ & $10\left(71.4 \%{ }^{\$}\right)$ & & \\
\hline Hypoglycemia, $\mathrm{n}(\%)$ & $2(8.7 \%)$ & & \\
\hline Hyperammonemia, $\mathrm{n}(\%)$ & $17(73.9 \%)$ & & \\
\hline High MCV, $\mathrm{n}(\%)$ & $14(60.9 \%)$ & & \\
\hline Gastrointestinal bleeding, $\mathrm{n}(\%)$ & $5(21.7 \%)$ & & \\
\hline Sepsis, $\mathrm{n}(\%)$ & $11(47.8 \%)$ & & \\
\hline Acute kidney injury, $\mathrm{n}(\%)$ & $16(69.6 \%)$ & & \\
\hline Chronic kidney disease, $\mathrm{n}(\%)$ & $2(8.7 \%)$ & & \\
\hline Liver cirrhosis, $\mathrm{n}(\%)$ & $9(39.1 \%)$ & & \\
\hline Hepatitis, $\mathrm{n}(\%)$ & $4(17.4 \%)$ & & \\
\hline Need of vasoactive support, $\mathrm{n}(\%)$ & $3(13.0 \%)$ & & \\
\hline Need of ventilator support, $\mathrm{n}(\%)$ & $2(8.7 \%)$ & & \\
\hline Need of renal replacement therapy, $\mathrm{n}(\%)$ & $1(4.3 \%)$ & & \\
\hline
\end{tabular}

Table 2. Clinical and laboratory characteristics. ${ }^{*}$ Based on 20 cases. ${ }^{\$}$ Based on 14 cases.

$($ Median $=17.8$ vs 14.6, P-value $=0.711)$ and APACH II $($ Median $=14.0$ vs 20.5, P-value $=0.176)$ score among these two sub-groups were not associated with the survival of AALA.

This study demonstrated an unusual finding that the survivors had lower nadir arterial blood $\mathrm{pH}$ compared to the non-survivors because of the small number of the non-survivors and metabolic acidosis presented in these two patient sub-groups was respiratory overcompensated by the use of mechanical ventilation. In addition, in comparison with non-survivors, survivors had lower APACHE II scores but higher peak blood lactate concentrations, though they did not reach statistical significance. Our results were inconsistent with the previous literature, which emphasized the potential seriousness of lactic acidosis and proved a strong association with increased mortality ${ }^{4-9}$. However, several case reports also showed a favorable outcome of patients with AALA who had similar high blood lactate level ${ }^{13-16}$. This finding might reflect the entity of AALA (type B lactic acidosis), which actually was not related to the balance of oxygen delivery and oxygen consumption. In our study, 10 patients with peak blood lactate level between 5 and $15 \mathrm{mmol} / \mathrm{L}$ did not have the need of vasoactive support during the treatment period of AALA. Moreover among 13 patients who had blood lactate level $>15 \mathrm{mmol} / \mathrm{L}$, only 3 of them needed vasoactive support.

Considering the relationship between the arterial blood $\mathrm{PH}$, bicarbonate concentration and blood lactate level among these 23 AALA patients, we found that some patients had a blood lactate concentration of $>5 \mathrm{mmol} / \mathrm{L}$ and the simultaneous arterial blood $\mathrm{PH}$ was $>7.35$. In fact, for those 4 patients whose blood lactate level $>10$ but $\leq 15 \mathrm{mmol} / \mathrm{L}$, their coexisting PH levels were 7.43, 7.47, 7.36, and 7.53, respectively. Arterial blood PH levels of $7.43,7.47$ and 7.53 were the combination of metabolic alkalosis and respiratory alkalosis while $\mathrm{PH}$ level of 7.36 was caused by mixed type of metabolic acidosis and respiratory alkalosis. One patient who was presenting blood lactate level $>15 \mathrm{mmol} / \mathrm{L}$ had coexisting $\mathrm{PH}$ of 7.37, which resulted from metabolic acidosis and respiratory alkalosis. Further discussions regarding the cause of respiratory alkalosis are warranted. Furthermore, the effect of mechanical ventilation was seen in patients due to their arterial blood PH levels of 7.35 and 7.53 instead of 7.37, 7.43 and 7.47. The presence of mixed type acid-base disturbances is common in critical care. Therefore, it is not feasible to predict the severity of lactic acidosis just by using the arterial blood $\mathrm{PH}$ and bicarbonate level.

The reason that alcohol should be considered is its important role in lactic acidosis diagnosed by the clinicians, although the reasons that the coexisting medical illnesses such as GI bleeding, sepsis, liver disease (liver cirrhosis, hepatitis), acute kidney injury and chronic kidney disease might affect the severity and clinical manifestations of lactic acidosis were not yet completely explained. Consider the contribution of AALA, 10 patients with peak blood lactate level between 5 and $15 \mathrm{mmol} / \mathrm{L}$ did not have the need of vasoactive support. Even in 13 patients with lactate level $>15 \mathrm{mmol} / \mathrm{L}$, only 3 patients needed vasoactive support. Of these AALA patients, no patients needed ventilator support when peak lactate level was $\leqq 10 \mathrm{mmol} / \mathrm{L}$, although 2 out of those 21 patients with blood lactate concentration $>10 \mathrm{mmol} / \mathrm{L}$ needed ventilator support. Similarly, no patients needed renal replacement therapy when blood lactate concentration was $\leqq 20 \mathrm{mmol} / \mathrm{L}$. However, 1 patient underwent renal replacement therapy while the peak blood lactate concentration was $35.8 \mathrm{mmol} / \mathrm{L}$.

According to previous study results regarding the discussion between the lactate clearance and patient prognosis ${ }^{17-26}$, our study reflected similar findings, i.e. in comparison with non-survivors, a better lactate clearance was found in survivors. The average lactate clearance rate per hour was $10.1 \% \pm 6.6 \%$ in survivors versus $3.6 \% \pm 1.1 \%$ in non-survivors. In addition, this study revealed an important finding that higher lactate clearance 


\begin{tabular}{|c|c|c|c|}
\hline Characteristics & $\begin{array}{c}\text { Survivors } \\
\text { Median/IRQ } \\
(\min \sim \max )\end{array}$ & $\begin{array}{c}\text { Non- survivors } \\
\text { Median }^{\$} \\
(\min \sim \max )\end{array}$ & $\mathbf{p}$ \\
\hline No. of patients & 21 & 2 & \\
\hline Age (years) & $51.0 / 10.5(32-78)$ & $45.5(40-51)$ & 0.561 \\
\hline Sex, male, n (\%) & $20(95.2 \%)$ & $2(100 \%)$ & 1.000 \\
\hline APACHE II score & $14.0 / 10(7-33)$ & $20.5(17-24)$ & 0.176 \\
\hline Peak lactate level (mmol/L) & $17.8 / 8.8(9.7-35.8)$ & $14.6(13.3-15.9)$ & 0.711 \\
\hline Lactate clearance rate per hour & $7.4 / 6.9(3.0-25.3)$ & $3.5(2.8-4.3)$ & $0.047^{*}$ \\
\hline Nadir arterial $\mathrm{pH}$ & $7.2 / 0.3(6.8-7.5)$ & $7.45(7.4-7.5)$ & $0.042^{*}$ \\
\hline Nadir arterial bicarbonate level $(\mathrm{mmol} / \mathrm{L})$ & $8.2 / 8.5(2.3-21.1)$ & $15.5(13.3-17.6)$ & 0.158 \\
\hline Detectable blood or urine ketone body, n (\%) & $15(78.9 \%)$ & $0(0 \%)$ & 0.250 \\
\hline Detectable serum alcohol level, n (\%) & $10(71.4 \%)$ & - & - \\
\hline Hypoglycemia, n (\%) & $2(9.5 \%)$ & $0(0 \%)$ & 1.000 \\
\hline Hyperammonemia, n (\%) & $15(71.4 \%)$ & $2(100 \%)$ & 1.000 \\
\hline High MCV, n (\%) & $13(61.9 \%)$ & $1(50 \%)$ & 1.000 \\
\hline Gastrointestinal bleeding, n (\%) & $4(19 \%)$ & $1(50 \%)$ & 0.395 \\
\hline Sepsis, n (\%) & $9(42.9 \%)$ & $2(100 \%)$ & 0.217 \\
\hline Acute kidney injury, n (\%) & $15(71.4 \%)$ & $1(50 \%)$ & 0.526 \\
\hline Chronic kidney disease, n (\%) & $2(9.5 \%)$ & $0(0 \%)$ & 1.000 \\
\hline Liver cirrhosis, $\mathrm{n}(\%)$ & $7(33.3 \%)$ & $2(100 \%)$ & 0.142 \\
\hline Hepatitis, n (\%) & $4(19 \%)$ & $0(0 \%)$ & 1.000 \\
\hline Vasoactive support, $\mathrm{n}(\%)$ & $3(14.3 \%)$ & $0(0 \%)$ & 1.000 \\
\hline Mechanical ventilation support, $\mathrm{n}(\%)$ & $0(0 \%)$ & $2(100 \%)$ & $<0.05^{*}$ \\
\hline Renal replacement therapy, n (\%) & $1(4.8 \%)$ & $0(100 \%)$ & 1.000 \\
\hline
\end{tabular}

Table 3. The comparison between survivors and non-survivors. Wilcoxon rank-sum tests were used for continuous variables and Fisher's exact tests was used for categorical variables. ${ }^{*} \mathrm{~A}$ statistically significant between-group difference $(\mathrm{P}<0.05)$. ${ }^{\$}$ Due to only 2 cases, no IQR performed.

rate and shorter lactate clearance time were found in patients with AALA and coexisting sepsis than those with solely sepsis-related lactic acidosis. In the report showed by Marty et al. ${ }^{26}$ the 24 hours lactate clearance in patients with severe sepsis or septic shock was $42 \% \pm 33 \%$ in survivors and $-17 \% \pm 76 \%$ in non-survivors, where the lactate clearance rate was $85.1 \% \pm 8.9 \%(66.2 \%$ to $93.3 \%)$ in patients with AALA along with sepsis in our study, and the average duration was 14 hours and 42 minutes.

There was a question of the serum alcohol level in patients presenting AALA. In this study, positive results were detected in 10 patients; however, the serum alcohol level was not an absolute indication for the diagnosis of AALA because either acute or chronic alcohol abuse, only whether alcohol intake was continued until the time of presentation mattered. Besides, all 23 AALA patients received thiamine therapy. Furthermore, our study showed that 14 patients had high MCV, which could be considered macrocytosis, and excessive alcohol intake itself or the combination with coexisting acute GI bleeding and liver disease could be the common causes and thus played important roles. In addition, among 17 patients who presented hyperammonemia, 11 of them were with liver diseases, which probably were related to excessive alcohol consumption as well.

There were several limitations in our study. First, this was a single center, retrospective observational study. Second, the patient's number was small. Third, AALA was defined as patients with alcohol abuse combining a blood lactate level $>5 \mathrm{mmol} / \mathrm{L}$, and the diagnosis had been considered by the clinicians in charge despite the serum alcohol level, even though the coexisting medical illnesses such as GI bleeding, sepsis, liver disease (liver cirrhosis, hepatitis), acute kidney injury and chronic kidney disease may also were contributed to lactic acidosis. Although it is not easy to calculate the real contribution of alcohol to the severity of lactic acidosis on AALA, the role of alcohol could not be underemphasized after consideration of 2 patients $(8.7 \%)$ presenting peak blood lactate level $<10 \mathrm{mmol} / \mathrm{L}$; other 21 patients $(91.3 \%)$ were with blood lactate concentration $>10 \mathrm{mmol} / \mathrm{L}$, including 8 patients $>10$ but $\leq 15 \mathrm{mmol} / \mathrm{L}$ as well as 5 patients $>15$ but $\leq 20 \mathrm{mmol} / \mathrm{L}$ and other 8 patients had a peak blood lactate level $>20 \mathrm{mmol} / \mathrm{L}$. Among these 23 patients, only 3 patients needed vasoactive support, ventilator support in 2 patients, and renal replacement therapy in 1 patient from blood lactate level $>5 \mathrm{mmol} / \mathrm{L}$ to $<5 \mathrm{mmol} / \mathrm{L}$.

To our knowledge, this study is the largest case series ever reported on critically ill patients with AALA, and the results show that the prognosis of AALA is better than the predicted outcome according to the severity of lactic acidosis. There were several case reports also showed a favorable outcome of patients with AALA who had similar high blood lactate level ${ }^{13-16}$. Even though the peak blood lactate concentration is not associated with the survival of AALA; however, survivors had better lactate clearance rate per hour than non-survivors and AALA patients with coexisting sepsis presenting higher lactate clearance rate and shorter lactate clearance time than those had solely sepsis-related lactic acidosis. 


\section{References}

1. Thomas, I. Ueber die Wirkung einiger narkotischer Stoffe auf die Blutqase, die Blutalkalescenz und die rothen Blutkorperchen. Arch Exp Pathol Pharmakol 41, 1-18 (1898).

2. Adrogue, H. J. \& Madias, N. E. Management of life-threatening acid-base disorders. Second of two parts. N Engl J Med 338, 107-111 (1998).

3. Stacpoole, P. W. Lactic acidosis. Endocrinol Metab Clin North Am 22, 221-245 (1993).

4. Weil, M. H. \& Afifi, A. A. Experimental and clinical studies on lactate and pyruvate as indicators of the severity of acute circulatory failure (shock). Circulation 41, 989-1001 (1970).

5. Cady, L. D. Jr. et al. Quantitation of severity of critical illness with special reference to blood lactate. Crit Care Med 1, 75-80 (1973).

6. Broder, G. \& Weil, M. H. Excess Lactate: An Index of Reversibility of Shock in Human Patients. Science 143, 1457-1459 (1964).

7. Kruse, J. A., M.K. \& Carlson, R. W. Definition of clinically significant lactic acidosis. Chest 100, 1008 (1987).

8. JA, K. Blood lactate and oxygen transport. Intensive Care World 4, 121-125 (1987).

9. Kruse, J. A., Zaidi, S. A. \& Carlson, R. W. Significance of blood lactate levels in critically ill patients with liver disease. Am J Med 83, 77-82 (1987).

10. Stacpoole, P. W. et al. Natural history and course of acquired lactic acidosis in adults. DCA-Lactic Acidosis Study Group. Am J Med 97, 47-54 (1994).

11. Gutierrez, G. \& Wulf, M. E. Lactic acidosis in sepsis: a commentary. Intensive Care Med 22, 6-16 (1996).

12. Luft, F. C. Lactic acidosis update for critical care clinicians. J Am Soc Nephrol 12 Suppl 17, S15-S19 (2001).

13. Ishii, K. et al. Two survival cases of alcoholic lactic acidosis complicated with diabetes mellitus and alcoholic liver disease. Alcohol Clin Exp Res 20, 387A-390A (1996).

14. Lien, D. \& Mader, T. J. Survival from profound alcohol-related lactic acidosis. J Emerg Med 17, 841-846 (1999).

15. Mussig, K., Schleicher, E. D., Haring, H. U. \& Riessen, R. Satisfactory outcome after severe ethanol-induced lactic acidosis and hypoglycemia. J Emerg Med 34, 337-338 (2008).

16. Shull, P. D. \& Rapoport, J. Life-threatening reversible acidosis caused by alcohol abuse. Nat Rev Nephrol 6, 555-559 (2010).

17. Manikis, P., Jankowski, S., Zhang, H., Kahn, R. J. \& Vincent, J. L. Correlation of serial blood lactate levels to organ failure and mortality after trauma. Am J Emerg Med 13, 619-622 (1995).

18. Bakker, J., Gris, P., Coffernils, M., Kahn, R. J. \& Vincent, J. L. Serial blood lactate levels can predict the development of multiple organ failure following septic shock. Am J Surg 171, 221-226 (1996).

19. McNelis, J. et al. Prolonged lactate clearance is associated with increased mortality in the surgical intensive care unit. Am J Surg 182, 481-485 (2001)

20. Nguyen, H. B. et al. Early lactate clearance is associated with improved outcome in severe sepsis and septic shock. Crit Care Med 32, 1637-1642 (2004).

21. Donnino, M. W. et al. Effective lactate clearance is associated with improved outcome in post-cardiac arrest patients. Resuscitation 75, 229-234 (2007).

22. Jones, A. E. et al. Lactate clearance vs central venous oxygen saturation as goals of early sepsis therapy: a randomized clinical trial. JAMA 303, 739-746 (2010).

23. Jansen, T. C. et al. Early lactate-guided therapy in intensive care unit patients: a multicenter, open-label, randomized controlled trial. Am J Respir Crit Care Med 182, 752-761 (2010).

24. Nguyen, H. B. et al. Outcome effectiveness of the severe sepsis resuscitation bundle with addition of lactate clearance as a bundle item: a multi-national evaluation. Crit Care 15, R229 (2011).

25. Nichol, A. et al. Dynamic lactate indices as predictors of outcome in critically ill patients. Crit Care 15, R242 (2011).

26. Marty, P. et al. Lactate clearance for death prediction in severe sepsis or septic shock patients during the first 24 hours in Intensive Care Unit: an observational study. Ann Intensive Care 3, 3 (2013).

27. Workgroup on Hypoglycemia, A.D.A. Defining and reporting hypoglycemia in diabetes: a report from the American Diabetes Association Workgroup on Hypoglycemia. Diabetes Care 28, 1245-1249 (2005).

\section{Acknowledgements}

We thank the staff of the department of intensive care medicine in Chi Mei Medical Center.

\section{Author Contributions}

C.-C.Y. contributed to the study design and helped draft the manuscript. K.-L.T. contributed to the study design, helped do the statistical analysis. S.-F.W. contributed to the study design, helped do the statistical analysis, and revised the manuscript. K.-S.C. contributed to the study design and revised the manuscript. All authors read and approved the final manuscript.

\section{Additional Information}

Competing financial interests: The authors declare no competing financial interests.

How to cite this article: Yang, C.-C. et al. Prognosis of alcohol-associated lactic acidosis in critically ill patients: an 8-year study. Sci. Rep. 6, 35368; doi: 10.1038/srep35368 (2016).

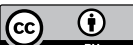

This work is licensed under a Creative Commons Attribution 4.0 International License. The images or other third party material in this article are included in the article's Creative Commons license, unless indicated otherwise in the credit line; if the material is not included under the Creative Commons license, users will need to obtain permission from the license holder to reproduce the material. To view a copy of this license, visit http://creativecommons.org/licenses/by/4.0/

(C) The Author(s) 2016 